\title{
Hoyeraal-Hreidarsson syndrome
}

INSERM

\section{Source}

INSERM. (1999). Orphanet: an online rare disease and orphan drug data base. HoyeraalHreidarsson syndrome. ORPHA:3322

Hoyeraal-Hreidarsson syndrome (HHS) is a very rare X-linked recessive disorder considered to be a severe variant of dyskeratosis congenita (see this term) characterized by intrauterine growth retardation, microcephaly, cerebellar hypoplasia, progressive combined immune deficiency and aplastic anemia. 